This is the final peer-reviewed accepted manuscript of:

Carmeli C., Fioresi R., Varadarajan V.S. (2019) The Exponential of Nilpotent Supergroups in the Theory of Harish-Chandra Representations. In: Nielsen F., Barbaresco F. (eds) Geometric Science of Information. GSI 2019. Lecture Notes in Computer Science, vol 11712. Springer, Cham

The final published version is available online at: https://doi.org/10.1007/978-3-030-269807_20

Rights / License:

The terms and conditions for the reuse of this version of the manuscript are specified in the publishing policy. For all terms of use and more information see the publisher's website.

This item was downloaded from IRIS Università di Bologna (https://cris.unibo.it/)

When citing, please refer to the published version. 


\title{
The exponential of nilpotent supergroups in the theory of Harish-Chandra representations *
}

\author{
C. Carmeli ${ }^{10000-0003-1660-3677]}$, R. Fioresi ${ }^{2[0000-0003-4075-7641]}$, and V. S. \\ Varadarajan $^{3[]}$ \\ 1 DIME, Università di Genova, Genova, Italy carmeli@dime.unige.it \\ 2 Dipartimento di Matematica, piazza Porta San Donato 5, University of Bologna, \\ 40126 Bologna, Italy \\ rita.fioresi@unibo.it \\ 3 Department of Mathematics, UCLA, Los Angeles, 90095-1555 CA, USA
}

\begin{abstract}
In this paper we discuss the exponential map in the case of nilpotent superalgebras. This provides global coordinates for nilpotent analytic supergroups, which are useful in the applications.
\end{abstract}

Keywords: Supergeometry · Lie Theory - Representation Theory.

\section{Introduction}

In supersymmetry, originally introduced by Berezin (see [3] and also [4], [21]), the concept of a Lie supergroup is central and it is indeed the search for extra symmetries of physical systems that let to the discovery of supersymmetry and later on of supergeometry (see [25], [26] and the comprehensive treatments [5], [24], [16] and the references within).

First, the notion of Lie superalgebra was introduced and only later on, there was a formalization of the notion of Lie and algebraic supergroup. The exponential map plays an eminent role and was originally introduced and studied by Koszul in [22] and later on the theory was further developed in [18].

In the algebraic setting, the recipe presented in [13], [14], [15] to construct an algebraic supergroup starting from a Lie superalgebra, uses in a implicit way the notion of exponential (see also [22], [6]).

In this paper we want to restrict our attention to a special case, which is however important in the applications, namely the case of a nilpotent analytic subsupergroup of an analytic complex matrix supergroup. We shall employ freely the language of Super Harish-Chandra pair (SCHP) introduced in [21] and developed by Koszul in his fundamental work [22].

In Sec. 2, we present the construction of the exponential, while in the subsequent sections we give applications, important in the study of the HarishChandra representations of supergroups (see [19], [7], [8]).

\footnotetext{
* Supported by funded project GHAIA (GA 777822).
} 
Acknowledgements. We are indebted to Prof. F. Gavarini for remarks and suggestions.

\section{The exponential map}

Let $\mathfrak{g}$ be a complex contragredient Lie superalgebra, $\mathfrak{g} \neq A(n, n), \mathfrak{g}_{1} \neq 0$; hence $\mathfrak{g}$ will be one in the following list of Lie superalgebras (see [20] Prop. 1.1):

$$
A(m, n) \text { with } m \neq n, B(m, n), C(n), D(m, n), D(2,1 ; \alpha), F(4), G(3)
$$

$\mathfrak{g}_{0}$ is either semisimple or with a one-dimensional center. Hence, by the ordinary theory, we know that the simply connected Lie group $\widetilde{G}, \mathfrak{g}_{0}=\operatorname{Lie}(\widetilde{G})$ is a matrix complex analytic and algebraic group. Then, the super Harish-Chandra pair SHCP $G=(\widetilde{G}, \mathfrak{g})$ (see [5] Ch. 11 and [12]) can be viewed either as a complex analytic or algebraic supergroup, via the theory of SHCP that establishes an equivalence of categories between the categories of analytic supergroups and SHCP (see [6]). We shall take the first point of view and regard $G$ as a complex analytic matrix supergroup, but later on we will also view $G$ as a pair $G=\left(\widetilde{G}, \mathcal{O}_{G}\right), \mathcal{O}_{G}$ a sheaf of superalgebras, (see $[6]$ ).

Fix $\mathfrak{h}$ a CSA of $\mathfrak{g}$ and fix $P$ a positive system. Let us define $\mathfrak{b}^{ \pm}$and $\mathfrak{n}^{ \pm}$the Borel and nilpotent subsuperalgebras:

$$
\mathfrak{g}=\mathfrak{h} \oplus \bigoplus_{\alpha \in \Delta} \mathfrak{g}_{\alpha}, \quad \mathfrak{b}^{ \pm}:=\mathfrak{h} \oplus \sum_{\alpha \in \pm P} \mathfrak{g}_{\alpha}, \quad \mathfrak{n}^{ \pm}:=\sum_{\alpha \in \pm P} \mathfrak{g}_{\alpha}
$$

We will call $B^{ \pm}$Borel subsupergroup and $N^{ \pm}$unipotent subsupergroup, their corresponding analytic Lie supergroups in $G$. In particular, $B^{ \pm}$and $N^{ \pm}$are connected and are algebraic subsupergroups of $G$. Let $A$ be the torus with $\operatorname{Lie}(A)=\mathfrak{h}$.

We want to define the exponential diffeomorphism: exp $: \mathfrak{n}^{-} \longrightarrow N^{-}$for the analytic supergroup $N^{-}$. To ease the notation we shall drop the index "-".

Our purpose in the construction of the exponential diffeomorphism is to obtain global coordinates on the nilpotent supergroup $N$; such coordinates are going to be essential for some important applications, (see [7], [8]).

We start with some general remarks on the functor of the $\Lambda$-points, we invite the reader to consult [1] and [2] for the complete details.

Let $M$ be a supermanifold. Instead of looking at the whole functor of points $M(\cdot):$ (smflds) $\rightarrow$ (sets), it is sometimes convenient to restrict the functor of points from the category (smflds) to the subcategory (spts) consisting of just the superpoints: $k^{0 \mid n}$. These are the supermanifolds $\left(\{*\}, \Lambda^{n}\right)$, where $\Lambda^{n}$ denotes the Grassmann algebra in $n$ generators over $k$. In this approach the set $M\left(k^{0 \mid n}\right)$ can be endowed with the structure of an ordinary manifold, but with some peculiarities. The tangent space at a point is a $\Lambda_{0}^{n}$-module and the change of coordinates induced by a change of coordinates in $M$ must have $\Lambda_{0}^{n}$-linear 
differential. These are called $\Lambda_{0}$-manifolds and we denote with $\left(\Lambda_{0}\right.$ mflds $)$ the corresponding category. The functor

$$
\text { (spts }) \rightarrow\left(\Lambda_{0} \text { mflds }\right) \quad k^{0 \mid n} \mapsto M\left(k^{0 \mid n}\right)
$$

is a full and faithful embedding (see [1] Sec. 4, Theorem 4.5). We notice that, if $V$ is a vector superspace, we have the identification $V\left(k^{0 \mid n}\right) \simeq\left(V \otimes \Lambda^{n}\right)_{0}$ and the previous result is known as the even rules principle (see also [10]).

Proposition 1. 1. If $G$ is a complex matrix supergroup as above, the $\Lambda_{0}$ manifold $G\left(k^{0 \mid n}\right)$ is a group object in the category $\left(\Lambda_{0} \mathrm{mflds}\right)$ and in particular it is an analytic Lie group. Similarly $\mathfrak{g}\left(k^{0 \mid n}\right)$ is an ordinary Lie algebra.

2. The ordinary exponential $\exp _{k^{0 \mid n}}: \mathfrak{g}\left(k^{0 \mid n}\right) \rightarrow G\left(k^{0 \mid n}\right)$ is a morphism of $\Lambda_{0}$ manifolds.

Proof. (1) is a simple check. As for (2), one can readily see that the differential of this map is $\Lambda_{0}$-linear and the correspondence $\mathfrak{g}\left(k^{0 \mid n}\right) \rightarrow G\left(k^{0 \mid n}\right)$ is functorial.

Since the functoriality property of exp in Prop. 1 (refer also to [1,2] for a thorough treatment of $\Lambda$-points), we can immediately define the exponential morphism for an analytic supergroup $G$.

Definition 1. Let $G$ and $\mathfrak{g}$ as above. We define the exponential map as the morphism of analytic supermanifolds given on the $\Lambda$-points as the ordinary exponential as in Prop 1 (2).

Proposition 2. Let $N$ be a nilpotent supergroup as above. Then the exponential morphism exp: $\mathfrak{n} \rightarrow N$ is a global superdiffeomorphism.

Proof. In case $N$ is a unipotent Lie supergroup as in (2), each $G(\Lambda)$ is also a unipotent Lie group and, by a classical result, each $\exp _{k^{0 \mid n}}$ is a diffeomorphism. Hence exp is a superdiffeomorphism.

\section{The nilpotent subsupergroup $N^{-}$}

In this section we give some applications of the global coordinates we have built in the previous section. Let $\Gamma=N^{-} A N^{+}$denote the big cell; it is the open analytic subsupermanifold $\Gamma=\left(\widetilde{N^{-A N^{+}}},\left.\mathcal{O}_{G}\right|_{N^{-A N^{+}}}\right)$of the analytic supergroup $G=$ $\left(\widetilde{G}, \mathcal{O}_{G}\right)$. We need some preliminary propositions.

Proposition 3. $N^{-}$is a section for $\Gamma \rightarrow \Gamma / B^{+}$, the left action of $A$ reads:

$$
A \times \Gamma / B^{+} \longrightarrow \Gamma / B^{+}, \quad\left(h, n B^{+}(A)\right) \mapsto h n h^{-1} B^{+}(A),
$$

where $n \in N^{ \pm}(T), h \in A(T), T \in(\text { smflds })_{\mathbb{C}}\left((\text { smflds })_{\mathbb{C}}\right.$ denoting the category of analytic supermanifolds). 
Proof. Since the big cell $\Gamma \subset G$ is right $B^{+}$-invariant and open, and the canonical projection $p: G \rightarrow G / B^{+}$is a submersion, we can define the open subsupermanifold of $G / B^{+}$:

$$
\Gamma / B^{+}:=\left(\widetilde{\Gamma / B^{+}},\left.\mathcal{O}_{G / B^{+}}\right|_{\Gamma / B^{+}}\right)
$$

We have a $N^{-}$equivariant diffeomorphism $N^{-} \longrightarrow \Gamma / B^{+}, n^{-} \mapsto n^{-} B^{+}(T)$, $n^{-} \in N^{-}(T), T \in(\text { smflds })_{\mathbb{C}}$. In fact, by the ordinary theory we have a diffeomorphisms of the underlying differentiable manifolds and the differential at the identity is an isomorphism: $\mathfrak{n}^{-} \cong \mathfrak{g} / \mathfrak{b}^{+}$.

Clearly $p^{-1}\left(\Gamma / B^{+}\right)=\Gamma$. We are going to construct a section $s: \Gamma / B^{+} \rightarrow \Gamma$. The local splitting $\gamma: N^{-} \times B^{+} \rightarrow \Gamma$ is an holomorphic morphism such that $\gamma^{*} \mathcal{O}_{\Gamma / B^{+}}=\mathcal{O}_{N^{-}} \otimes 1$. Hence we have an isomorphism $N^{-} \rightarrow \Gamma / B^{+}$given by the composition of the "canonical" embedding $i: N^{-} \hookrightarrow N^{-} \times B^{+}$with $\gamma$ and $p$ (which is essentially the same as considering $p \circ \gamma_{\left.\right|_{N-x\{e\}}}$ ). Its inverse is the required section.

Proposition 4. The (ordinary) torus A normalizes $N^{ \pm}$.

Proof. We give the proof for $N^{+}=N$. We want to prove that the conjugation

$$
\operatorname{conj}(a): G \rightarrow G \quad \operatorname{conj}(a)=\ell_{a^{-1}} \circ r_{a}, \quad a \in \widetilde{A}
$$

stabilizes $N$. Since $N$ is connected and the exponential map exp: $\mathfrak{n} \rightarrow N$ is surjective it is enough to prove that $(d \operatorname{conj}(a))_{1}(\mathfrak{n}) \subseteq \mathfrak{n}$ We know from the infinitesimal theory that $\operatorname{ad}(\mathfrak{h})(\mathfrak{n})=\mathfrak{n}$. Hence, we have

$$
\operatorname{Ad}\left(e^{t X}\right) Y=e^{\operatorname{tad} X}(Y) \quad \forall X \in \mathfrak{h}, Y \in \mathfrak{n}
$$

so that $\operatorname{Ad}\left(e^{t X}\right) \mathfrak{n}=\mathfrak{n}$. Since the exponential map of an abelian connected Lie group is surjective we have that $\operatorname{Ad}(A) \mathfrak{n}=\mathfrak{n}$.

By the simply connectedness of $\widetilde{N}$, we get a map $\widetilde{\operatorname{conj}(a)}: \widetilde{N} \rightarrow \widetilde{N}$. It is easy to check that the pair

$$
\operatorname{Ad}(a): \mathfrak{n} \rightarrow \mathfrak{n} \quad \widetilde{\operatorname{conj}(a)}: \widetilde{N} \rightarrow \widetilde{N}
$$

is a SHCP morphism: $(\widetilde{\operatorname{conj}(a)}, \operatorname{Ad}(a)):(\widetilde{N}, \mathfrak{n}) \longrightarrow(\widetilde{G}, \mathfrak{g})$, so that, by the equivalence of categories between analytic SHCP and analytic supergroups, we have a morphism of super Lie groups $N \rightarrow G$. Since its differential coincides with the differential of $\operatorname{conj}(a): N \longrightarrow G$ and the reduced maps are the same, the two morphisms coincide, hence $\operatorname{conj}(a) N=N$.

Let us fix a character $\chi: A \longrightarrow \mathbb{C}^{\times}$of the ordinary torus, that we can trivially extend to a character (still denoted by $\chi$ ) of the supergroup $B^{+}$. Define:

$$
L^{\chi}(\Gamma):=\left\{f \in \mathcal{O}_{G}(\Gamma) \mid f(g b)=\chi(b)^{-1} f(g)\right\}
$$


We can geometrically view this superspace as the superspace of sections of the line bundle uniquely associated with $\chi$. This superspace is the key for the construction of the infinite dimensional representations of real forms of the analytic supergroup $G$ (see [7], [8] for more details). The actions that we are going to describe are absolutely essential for the realization of such representations.

Since $A$ acts on $N^{-}$by conjugation (see Prop. 4), we have a global action of $A$ on $\Gamma$ defined as:

$$
a \cdot\left(n^{-} b^{+}\right)=\left(a n^{-} a^{-1}\right) b^{+}, \quad a \in \widetilde{A}, n^{-} \in N^{-}(T), b^{+} \in B^{+}(T) .
$$

Since $A$ also acts on $B^{+}$by left translation, we can define the left action of $A$ on $\Gamma$ as:

$$
a \cdot\left(n^{-} b^{+}\right)=\left(a n^{-} a^{-1}\right) a \cdot b^{+} .
$$

Both actions commute with right translations by $B^{+}$and hence define representations of $A$ on $L^{\chi}(\Gamma)$

$$
i, \ell: A \times L^{\chi}(\Gamma) \rightarrow L^{\chi}(\Gamma)
$$

where:

$$
i_{a}(f)\left(n^{-} b^{+}\right)=f\left(\left(a^{-1} n^{-} a\right) b^{+}\right), \quad \ell_{a}(f)\left(n^{-} b^{+}\right)=f\left(\left(a^{-1} n^{-} a\right) a^{-1} b^{+}\right)
$$

and $a \in \widetilde{A}, n^{-} \in N^{-}(T), b^{+} \in B^{+}(T), f \in L^{\chi}(\Gamma)$.

Let $t_{\alpha}$ denote the global homogeneous exponential coordinates on $N^{-}$obtained by Prop. 2.

Lemma 1. Let the notation be as above. Then

1. $\ell_{a} f=\chi(a)\left(i_{a} f\right)$

2. $i_{a} t_{\alpha}=\chi_{\alpha}(a) t_{\alpha} \quad \forall a \in \widetilde{A}$

where $\chi_{\alpha}$ is the character of the maximal torus $A$ obtained by exponentiating the root $\alpha \in \mathfrak{h}^{*}$.

Proof. (1) follows immediately from the definitions. For (2) let $n=\exp \left(\sum_{\beta \in P}\right.$ $\left.y_{\beta} X_{-\beta}\right)$ be an element in $N^{-}$, then the result comes from the following formal calculation in the exponential global coordinates:

$$
\begin{aligned}
t_{\alpha}\left(a^{-1} n a\right) & =t_{\alpha}\left(\exp \left(\sum_{\beta \in P} y_{\beta} A d(a) X_{-\beta}\right)\right)=t_{\alpha}\left(\exp \left(\sum_{\beta \in P} y_{\beta} \chi_{\beta}(a) X_{-\beta}\right)\right. \\
& =\chi_{\alpha}(a) t_{\alpha}(n), \quad a \in \widetilde{A}, y_{\beta} \in \mathbb{C}
\end{aligned}
$$

\section{The action of $\mathcal{U}(\mathfrak{g})$ and $G$ on $L^{\chi}(\Gamma)$}

Now we want to use the theory developed so far and extend the action of the maximal torus $A \subset G$ to an action of the whole group on $L^{\chi}(\Gamma)$. We start by defining the natural action of $\mathcal{U}(\mathfrak{g})$ on the holomorphic functions on any neighbourhood $W$ of the identity of the supergroup $G$. 
Definition 2. Let $W \subset G$ be an open neighbourhood of the identity $1_{G}$ in $G$. There are two well defined actions of $\mathfrak{g}$, hence of $\mathcal{U}(\mathfrak{g})$, on $\mathcal{O}_{G}(W)$, that read as follows:

$$
\ell(X) f=(-X \otimes 1) \mu^{*}(f), \quad \partial(X) f=(1 \otimes X) \mu^{*}(f), \quad X \in \mathfrak{g}
$$

The actions $\ell$ and $\partial$ commute with each other. Moreover, if $\widetilde{U}$ is open in $\widetilde{G / B^{+}}$, then $\ell$ is a well defined action on $L^{\chi}(\widetilde{U})$.

We now want to show that the natural action $\ell$ of $\mathcal{U}(\mathfrak{g})$ on $L^{\chi}\left(N^{-} B^{+}\right)$preserves the polynomial sections on $\widetilde{N^{-}}$. For this we need some preliminary notation. Since $\mathfrak{g}=\mathfrak{n}^{-} \oplus \mathfrak{b}^{+}$, if we fix bases of $\mathfrak{n}^{-}$and $\mathfrak{b}^{+}$, by the PBW (Poincaré Birkhoff Witt) theorem any $X \in \mathcal{U}(\mathfrak{g})$ can be written as

$$
X=\sum_{I, J} c_{I J}(X) \mathrm{B}_{I} \mathrm{~N}_{I}, \quad \mathrm{~B}_{I} \in \mathcal{U}\left(\mathfrak{b}^{+}\right), \mathrm{N}_{I} \in \mathcal{U}\left(\mathfrak{n}^{-}\right)
$$

Lemma 2. Let $\phi \in \mathcal{O}_{G}\left(N^{-} B^{+}\right)$. In the SHCP notation, $\phi$ is in $L^{\chi}\left(N^{-} B^{+}\right)$if and only if

$$
\phi(X)(n b)=\widetilde{\chi}(b)^{-1} \sum_{I J} c_{I J}(b . X) \lambda\left(\overline{\mathrm{B}}_{I}\right) \phi\left(\mathrm{N}_{J}\right)(n), \quad X \in \mathcal{U}(\mathfrak{g}), \quad \lambda=d \chi
$$

where b.X is the adjoint action of $b \in \widetilde{B^{+}}$on $\mathcal{U}(\mathfrak{g})$ and as usual $\overline{\mathrm{B}}_{I}$ denotes the antipode of $\mathrm{B}_{I}$ in the Hopf superalgebra $\mathcal{U}(\mathfrak{g})$.

Proof. By the very definition we have $\phi \in L^{\chi}\left(N^{-} B^{+}\right)$if

1. $r_{b}^{*} \phi=\widetilde{\chi}(b)^{-1} \phi, b \in \widetilde{B^{+}}$

2. $D_{Y}^{L}(\phi)=\lambda(\bar{Y}) \phi,\left.\lambda\right|_{\mathfrak{g}_{0}}=d \widetilde{\chi}$.

where as usual $\tilde{\chi}$ denotes the reduced morphism. The result comes with a calculation.

Notice that once the lemma is established, if $p$ is a polynomial in the global coordinates of $N^{-}$, we can define $p^{\sim} \in L^{\chi}\left(N^{-} B^{+}\right)$as:

$$
p^{\sim}(X)(n b)=\widetilde{\chi}(b)^{-1} \sum_{I J} c_{I J}(b . X) \lambda\left(\overline{\mathrm{B}^{+}}\right) p\left(\mathrm{~N}_{J}\right)(n)
$$

Vice-versa we can recover $p$ from $p^{\sim}$ restricting to $N^{-}$. In the language of SHCP this amounts to two restrictions: we impose $b=1$ and $X \in \mathcal{U}\left(\mathfrak{n}^{-}\right)$. We shall denote the set of such $p^{\sim}$ with $\mathcal{P}^{\sim}$.

Proposition 5. The actions $\ell$ of $\mathcal{U}(\mathfrak{g})$ on $L^{\chi}(\widetilde{U}), p^{-1}(\widetilde{U}) \subset \Gamma$ leave $\mathcal{P}^{\sim}$ invariant. 
Proof. We need to show that, given $Z \in \mathcal{U}(\mathfrak{g})$ and $X \in \mathcal{U}\left(\mathfrak{n}^{-}\right),\left(\left.D_{Z}^{R} p^{\sim}\right|_{N^{-}}\right)(X)$ is a polynomial section. We have (see [5] Sec. 7.4):

$$
\left(D_{Z}^{R} p^{\sim}\right)(X)(g)=(-1)^{|Z||p|}\left[p^{\sim}\left(\left(g^{-1} . Z\right) X\right)\right](g)
$$

Hence if $n \in N^{-}$, we have:

$$
\begin{aligned}
\left(D_{Z}^{R} p^{\sim}\right)(X)(n) & =(-1)^{|Z||p|}\left[p^{\sim}\left(\left(n^{-1} . Z\right) X\right)\right](n) \\
& =(-1)^{|Z||p|} \sum_{I J} c_{I J}\left(\left(n^{-1} . Z\right) X\right)\left[\lambda\left(\overline{\mathrm{B}}_{I}\right) p^{\sim}\left(\mathrm{N}_{J}\right)\right](n)
\end{aligned}
$$

where $\mathrm{B}_{I}$ and $\mathrm{N}_{J}$ are obtained as in (5) applied to $\left(n^{-1} . Z\right) X$. The last equality is true by Lemma 2 .

Once this is established, we have the following result.

Theorem 1. There is a non-singular $\mathcal{U}(\mathfrak{g})$ invariant pairing between $\mathcal{P}^{\sim}$ and the Verma module $V_{\lambda}$ :

$$
\langle,\rangle: \mathcal{P}^{\sim} \times \mathcal{U}(\mathfrak{g}) \longrightarrow \mathbb{C}, \quad\langle f, u\rangle:=(-1)^{|u||f|}(\partial(u) f)\left(1_{G}\right)
$$

Proof. In order for $\langle$,$\rangle to be a \mathcal{U}(\mathfrak{g})$ invariant pairing, we need to verify:

$$
\langle\ell(c) f, u\rangle=\left\langle f,(-1)^{|f||c|} c^{T} u\right\rangle, \quad c, u \in \mathcal{U}(\mathfrak{g}), f \in \mathcal{P}^{\sim}
$$

where $(\cdot)^{T}$ denotes the antiautomorphism of $\mathcal{U}(\mathfrak{g})$ induced by $X \mapsto-X$ with $X \in \mathfrak{g}$. This is just a check.

Now let $\mathfrak{g}_{r}$ be a real form of $\mathfrak{g}$ and define the real supergroup $G_{r}=\left(\widetilde{G_{r}}, \mathfrak{g}_{r}\right)$, where $\widetilde{G_{r}}$ is a real form of $\widetilde{G}, \operatorname{Lie}\left(\widetilde{G_{r}}\right)=\mathfrak{g}_{r, 0}$. Since $\mathfrak{g}_{r}+\mathfrak{b}^{+}=\mathfrak{g}$ as real superalgebras (see [9], Iwasawa decomposition), we have that $S:=G_{r} B^{+}$is an open subsupermanifold of $G$.

Theorem 2. Assume $L^{\chi}(S) \neq 0$ modulo $J$ the submodule generated by the odd part. Then $L^{\chi}(S)$ contains an element $\psi$ which is an analytic continuation of $1 \sim$ and

$$
\ell(\mathcal{U}(\mathfrak{g})) \psi=\mathcal{P}^{\sim} \cong \pi_{-\lambda}
$$

where $\pi_{-\lambda}$ the irreducible representation with lowest weight $-\lambda$. Furthermore $L^{\chi}(S)$ carries a $G_{r}$ representation defined as:

$$
\begin{cases}(g \cdot f)=l_{g^{-1}}^{*} f & g \in \widetilde{G_{r}} \\ X . f=D \frac{R}{X} f & X \in \mathfrak{g}_{\mathbb{C}}\end{cases}
$$

where, as usual, $\bar{X}$ is the antipode of $X \in \mathcal{U}(\mathfrak{g})$.

Proof. Direct check.

The closure of $\ell(\mathcal{U}(\mathfrak{g})) \psi$ in $L^{\chi}(S)$, with a Fréchet superspace structure is a Harish-Chandra representation, with $\ell(\mathcal{U}(\mathfrak{g})) \psi$ as its $K_{r}$ finite part, where $K_{r}$ is the supergroup corresponding to the subalgebra $\mathfrak{k}_{r}$ in the Cartan decomposition of $\mathfrak{g}_{r}$ (see [9]). The proof of these facts is non trivial, we invite the reader to see $[7],[8]$. 


\section{References}

1. L. Balduzzi, C. Carmeli, R. Fioresi, The local functors of points of supermanifolds. Exp. Math. 28 (2010), no. 3, 201-217.

2. L. Balduzzi, C. Carmeli, R. Fioresi A comparison of the functors of points of supermanifolds. J. Algebra Appl. 12 (2013), no. 3, 125-152, 41 pp.

3. F. A. Berezin, Introduction to superanalysis. D. Reidel Pub., Holland, 1987.

4. F. A. Berezin, D. Leites, Supermanifolds, Dokl. Akad. Nauk SSSR, Vol. 224, no. 3, 505-508, 1975.

5. C. Carmeli, L. Caston, R. Fioresi, Mathematical Foundation of Supersymmetry, EMS Ser. Lect. Math., European Math. Soc., Zurich, 2011.

6. C. Carmeli, R. Fioresi, Super Distributions, Analytic and Algebraic Super HarishChandra pairs, Pac. J. Math., vol. 263, p. 29-51, 2013.

7. C. Carmeli, R. Fioresi, V.S. Varadarajan, Super Bundles, Universe, (2018), 4(3), 46.

8. C. Carmeli, R. Fioresi, V. S. Varadarajan Highest weight Harish-Chandra supermodules and their geometric realizations., Trasf. Groups, preprint, 2019.

9. M. K. Chuah, Fioresi R., Hermitian real forms of contragredient Lie superalgebras, J. Algebra, Vol. 437, 161-176, 2015.

10. P. Deligne, J. Morgan, Notes on supersymmetry (following J. Bernstein), in: "Quantum fields and strings. A course for mathematicians", Vol. 1, AMS, 1999.

11. R. Fioresi, Compact forms of complex Lie supergroups. J. Pure Appl. Alg., vol. 218, p. 228-236, 2014.

12. R. Fioresi, Smoothness of Algebraic Supervarieties and Supergroups, Pac. J. Math., 234, 295-310, 2008.

13. R. Fioresi, F. Gavarini, Chevalley Supergroups, AMS Memoirs, vol. 215, 1-64, 2012.

14. R. Fioresi, F. Gavarini, On Algebraic Supergroups with Lie superalgebras of classical type, J. Lie Theory, vol. 23, p. 143-158, 2013.

15. R. Fioresi, F. Gavarini, On the construction of Chevalley supergroups. Lecture Notes in Math., 2027, Springer, Heidelberg, 2011.

16. R. Fioresi, M. A. Lledo The Minkowski and Conformal Superspaces: The Classical and Quantum Descriptions, World Scientific Publishing, 2015.

17. R. Fioresi, M. A. Lledo, V. S. Varadarajan The Minkowski and conformal superspaces, J. Math. Phys., 48, 113505, 2007.

18. S. Garnier, T. Wurzbacher, Integration of vector fields on smooth and holomorphic supermanifolds, Documenta Mathematica, 18, (2013), 519-545.

19. Harish-Chandra, Representations of semi-simple Lie groups IV, V, VI. Amer. J. Math. no. 77, 743-777 (1955); no. 78, 1-41 and 564-628, (1956).

20. V. G. Kac, Lie superalgebras, Adv. Math. 26 (1977), 8-26.

21. B. Kostant. Graded manifolds, graded Lie theory, and prequantization. Lecture Notes in Math., Vol. 570, Springer, Berlin, 1977.

22. J.-L., Koszul, Graded manifolds and graded Lie algebras, Proceedings of the international meeting on geometry and physics (Florence, 1982), 71-84, Pitagora, Bologna, 1982.

23. D. A. Leites, Introduction to the theory of supermanifolds, Russian Math. Surveys 35: 1 (1980), 1-64.

24. V. S. Varadarajan, Supersymmetry for mathematicians: an introduction, Courant Lecture Notes 1, AMS, 2004.

25. D.V. Volkov, V.P. Akulov, Is the neutrino a Goldstone particle?, Phys. Lett. B 46 (1973) 109. 
26. J. Wess, B. Zumino. Supergauge transformations in four dimensions. Nucl. Phys. B, 70, (1974), 39-50. 